\title{
Description and Analysis of Cytokine Storm in Registered COVID-19 Clinical Trials: A Systematic Review
}

\author{
Khalid Eljaaly ${ }^{1,2, *}+{ }^{(D}$, Husam Malibary ${ }^{3}$, Shaimaa Alsulami ${ }^{4,5}$, Muradi Albanji ${ }^{4}$, Mazen Badawi ${ }^{6}$ \\ and Jaffar A. Al-Tawfiq $7,8,9$ (D)
}

1 Faculty of Pharmacy, Department of Pharmacy Practice, King Abdulaziz University, Jeddah 21589, Saudi Arabia

2 College of Pharmacy, Department of Pharmacy Practice and Science, King Abdulaziz University, Tucson, AZ 85724, USA

3 Allergy and Clinical Immunology Division, Faculty of Medicine, Department of Internal Medicine, King Abdulaziz University, Jeddah 21589, Saudi Arabia; hmmalibary@kau.edu.sa

4 Department of Pharmacy, King Abdulaziz University Hospital, Jeddah 21589, Saudi Arabia; alsulamish3@nhga.med.sa (S.A.); Albanji@kau.edu.sa (M.A.)

5 Department of Pharmacy, King Fahad Armed Forces Hospital, Jeddah 21159, Saudi Arabia

6 Infectious Disease Division, Faculty of Medicine, Department of Internal Medicine, King Abdulaziz University, Jeddah 21589, Saudi Arabia; mabadawi@kau.edu.sa

7 Infectious Disease Unit, Specialty Internal Medicine, Johns Hopkins Aramco Healthcare, Dhahran 31311, Saudi Arabia; jaffar.tawfiq@jhah.com

8 Infectious Disease Division, Department of Medicine, Indiana University School of Medicine, Indianapolis, IN 46202, USA

updates

Citation: Eljaaly, K.; Malibary, H.; Alsulami, S.; Albanji, M.; Badawi, M.; Al-Tawfiq, J.A. Description and Analysis of Cytokine Storm in Registered COVID-19 Clinical Trials: A Systematic Review. Pathogens 2021, 10, 692. https://doi.org/10.3390/ pathogens 10060692

Academic Editors: Jaume Ordi,

Natalia Rakislova and Lawrence S. Young

Received: 22 April 2021

Accepted: 31 May 2021

Published: 2 June 2021

Publisher's Note: MDPI stays neutral with regard to jurisdictional claims in published maps and institutional affiliations.

Copyright: (c) 2021 by the authors. Licensee MDPI, Basel, Switzerland. This article is an open access article distributed under the terms and conditions of the Creative Commons Attribution (CC BY) license (https:// creativecommons.org/licenses/by/ $4.0 /)$.
9 Infectious Disease Division, Department of Medicine, Johns Hopkins University, Baltimore, MD 21287, USA

* Correspondence: keljaaly@kau.edu.sa; Tel.: +1-(857)272-2994

+ Twitter: http://twitter.com/khalideljaaly.

\begin{abstract}
The purpose of this systematic review was to describe the characteristics of clinical trials that focused on COVID-19 patients with cytokine release syndrome (CRS) and the variability in CRS definitions. Two authors independently searched three clinical trial registries and included interventional clinical trials on COVID-19 hospitalized patients that required at least one elevated inflammatory biomarker. Relevant data, including the type and cutoff of the measured biomarker, oxygen/respiratory criteria, fever, radiologic criteria, and medications, were summarized. A total of 47 clinical trials were included. The included studies considered the following criteria: oxygen/respiratory criteria in 42 trials (89\%), radiologic criteria in 29 trials (62\%), and fever in 6 trials (18\%). Serum ferritin was measured in 35 trials (74\%), CRP in 34 trials (72\%), D-dimer in 26 trials (55\%), LDH in 24 trials (51\%), lymphocyte count in 14 trials (30\%), and IL-6 in 8 trials (17\%). The cutoff values were variable for the included biomarkers. The most commonly used medications were tocilizumab, in 15 trials (32\%), and anakinra in 10 trials (24.4\%). This systematic review found high variability in CRS definitions and associated biomarker cutoff values in COVID-19 clinical trials. We call for a standardized definition of CRS, especially in COVID-19 patients.
\end{abstract}

Keywords: cytokine storm; cytokine release syndrome; definition; systematic review

\section{Introduction}

Coronavirus disease 2019 (COVID-19) has rapidly spread across continents and became a global pandemic [1]. Patients with COVID-19 frequently experience pneumonia and may develop acute respiratory distress syndrome (ARDS). ARDS is characterized by bilateral lung infiltrates and severe progressive hypoxemia, and patients need to be admitted to the critical care unit and receive respiratory support [2,3]. ARDS is a rapidly progressive condition and is associated with a high mortality rate in COVID-19 patients. Severe acute respiratory syndrome coronavirus 2 (SARS-CoV-2) seems to cause comparable immunopathogenic features as those seen in SARS-CoV and MERS-CoV infections [2]. 
ARDS is one of the features of cytokine release syndrome (CRS), also known as cytokine storm, and its exact mechanism is still not well understood [4]. Hyperactive immune responses and cytokine overproduction have been associated with the pathogenesis of infectious and non-infectious diseases [4]. The term CRS captured the attention not only of the scientific publications but also of the media. CRS is a systemic inflammatory response mediated by the overproduction of pro-inflammatory cytokines, which can be stimulated by different factors, including infections and certain medications $[5,6]$. CRS is a major cause of morbidity in patients infected with SARS-CoV and MERS-CoV [7]. CRS was first described as an adverse reaction of the solid organ immunosuppressive medication, an anti-T cell antibody muromonab-CD 3 (OKT3), in the early 1990s [8]. Although the incidence of CRS with conventional monoclonal antibodies is relatively low, it is relatively high with $\mathrm{T}$ cell-engaging cancer immunotherapy [9]. Several clinical factors are associated with the severity of CRS following chimeric antigen receptor (CAR) T cell therapy [10]. Its severity ranges from mild symptoms, such as flu-like symptoms, fever, fatigue, headache, rash, arthralgia, and myalgia, to severe life-threatening reactions characterized by hypotension and high-grade fever. CRS can also progress to an uncontrolled systemic inflammatory response with vasopressor-requiring circulatory shock, vascular leakage, disseminated intravascular coagulation, and multi-organ system failure. CRS respiratory symptoms are common and can be mild as cough and tachypnea. However, it may progress to ARDS with dyspnea, hypoxia, and bilateral opacities on a chest $x$-ray requiring mechanical ventilation. Life-threatening CRS associated with mechanical ventilation is not caused by the respiratory disease mechanism alone but also the inability to protect the airway due to secondary neurotoxicity [11]. Multi-organ dysfunction in severe CRS includes renal failure, cardiac dysfunction with reduced ejection fraction, and vascular leakage with peripheral and pulmonary edema. Laboratory abnormalities associated with CRS include high inflammatory biomarkers. Severe CRS might be associated with laboratory abnormalities resembling hemophagocytic lymphohistiocytosis (HLH) or macrophage activation syndrome (MAS) [12]. It has been suggested that severe COVID-19 patients should be screened for hyperinflammation by using laboratory biomarkers and HScore. Hscore is usually used to generate a probability for the presence of secondary HLH, and to recognize the subgroup of patients for whom immunosuppression could decrease ARDS and mortality [13]. In depth understanding of the clinical picture and the underlining pathophysiology of CRS is crucial to establish early and effective management of this syndrome. There is no consensus on the definition of CRS and associated changes in inflammatory biomarkers $[4,6]$. Therefore, the objective of this systematic review was to describe the variability in identifying patients with cytokine storm in clinical trials of COVID-19 patients and the relevant characteristics of these trials.

\section{Materials and Methods}

Two investigators independently screened the ClinicalTrials.gov, European Union Clinical Trials Register, and World Health Organization International Clinical Trials Registry Platform between 1 November 2019 and 23 October 2020. The search strategy is provided in Table 1. We included interventional clinical trials on COVID-19 hospitalized patients that required at least one elevated inflammatory biomarker (CRP, ferritin, D-dimer, LDH, IL-6, or lymphocyte count) in their inclusion criteria and mentioned any of the following terminologies "cytokine storm", "cytokine storm syndrome", "cytokine release syndrome", "hyperinflammation", "macrophage activation syndrome", "immune dysregulation", or "hemophagocytic lymphohistiocytosis". From each clinical trial, the following data were extracted: registration number, recruitment country, whether included patients were adults or pediatrics, studied medications, the type and cutoff of measured biomarkers, whether fever, oxygen/respiratory, and/or radiologic criteria were used in addition to the biomarkers. The unit of each biomarker was converted to the one most commonly used unit for convenience to allow for numerical comparison ( $\mu \mathrm{g} / \mathrm{L}$ for ferritin, $\mathrm{mg} / \mathrm{L}$ for CRP, $\mathrm{ng} / \mathrm{mL}$ for D-dimer, cells / $\mu \mathrm{L}$ for lymphocytes, IU / $\mathrm{L}$ for $\mathrm{LDH}$, and $\mathrm{pg} / \mathrm{mL}$ for IL-6). 
Table 1. Search Strategy.

\begin{tabular}{|c|c|}
\hline Database & Search Strategy \\
\hline ClinicalTrials.gov & $\begin{array}{l}\text { COVID-19 AND ("cytokine" OR “hyperinflammation" OR "macrophage activation } \\
\text { syndrome" OR "immune dysregulation" OR “hemophagocytic lymphohistiocytosis"). } \\
\text { Restricted to interventional studies (clinical trials). }\end{array}$ \\
\hline EU Clinical Trial Register & $\begin{array}{l}\text { COVID-19 AND ("cytokine" OR "hyperinflammation" OR "macrophage activation } \\
\text { syndrome" OR "immune dysregulation" OR "hemophagocytic lymphohistiocytosis"). }\end{array}$ \\
\hline $\begin{array}{l}\text { WHO International Clinical Trials } \\
\text { Registry Platform }\end{array}$ & $\begin{array}{l}\text { COVID-19 AND cytokine OR COVID-19 AND hyperinflammation OR COVID-19 } \\
\text { AND macrophage activation syndrome OR COVID-19 AND immune dysregulation } \\
\text { OR COVID-19 AND hemophagocytic lymphohistiocytosis. }\end{array}$ \\
\hline
\end{tabular}

\section{Results}

Out of 413 clinical trials screened, a total of 47 trials were included (Figure 1). The study characteristics of these studies are presented in Tables 2 and A1, and a summary of the included parameters in the definition of the different studies is provided in Table 3. A total of 26 trials (55.3\%) were in Europe, $11(23.4 \%)$ were in North America, $2(4.2 \%)$ were in South America, $6(12.8 \%)$ were in Asia, and $2(4.2 \%)$ were multicontinental. Almost all clinical trials included only adult patients and one study included patients $\geq 12$ years, in the United States only (NCT04362813). The most commonly studied medications were as follows: tocilizumab (15 trials; $32 \%$ ), anakinra (10 trials; $24.4 \%)$, corticosteroids (4 trials; $8.5 \%$ ), sarilumab (4 trials; $8.5 \%$ ), clazakizumab (3 trials; $6.3 \%$ ), ruxolitinib ( 3 trials; $6.3 \%$ ), emapalumab ( 2 trials; $4.2 \%$ ), and siltuximab ( 2 trials; $4.2 \%$ ). The following interventions were used in one study each: canakinumab, gimsilumab, itolizumab, mavrilimumab, cytokine adsorption, CytoSorb, hyperbaric oxygen, radiotherapy, collagen-polyvinylpyrrolidone, therapeutic plasma exchange, trimethoprim-sulfamethoxazole, reparixin, losmapimod, defibrotide, dornase alfa, etoposide, pyridostigmine, and zilucoplan. The inclusion criteria that were used in these studies were as follows: oxygen/respiratory criteria in 42 trials $(89 \%)$, radiologic criteria in 27 trials $(62 \%)$, and fever in 6 trials $(19 \%)$. Other used clinical criteria were mainly shock and organ dysfunction in four trials (NCT04424056, NCT04339712, DRKS00021447, NCT04366232). Only one trial included inflammatory biomarkers without clinical or radiological criteria (NCT04423042).

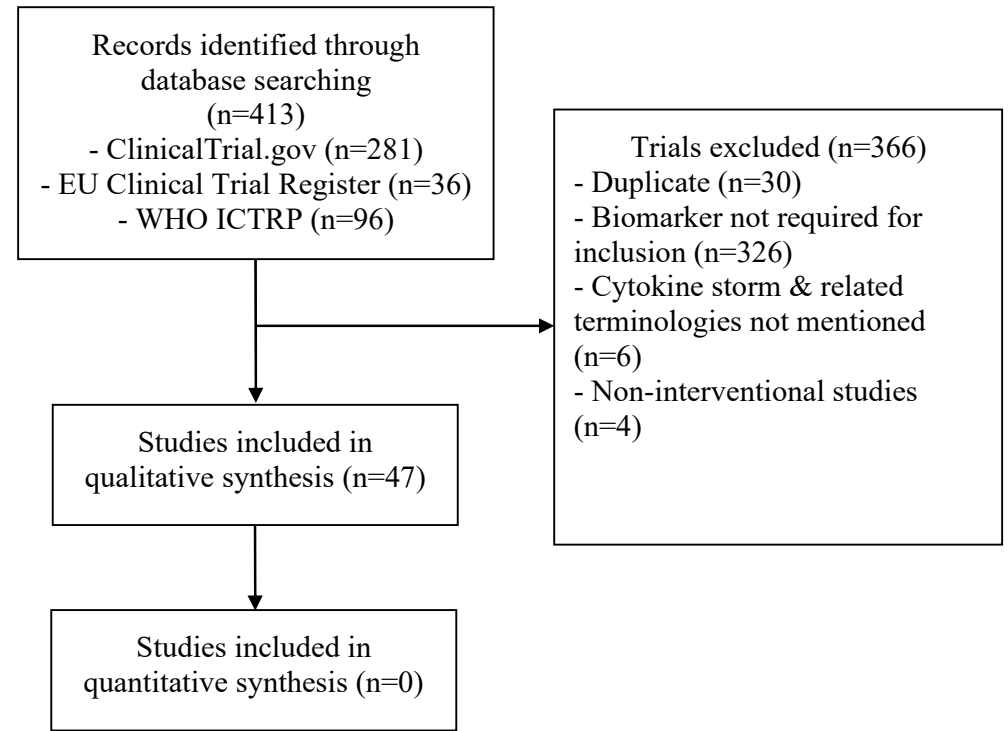

Figure 1. Flow diagram of the study selection process. 
Table 2. Summary and characteristics of the included studies describing cytokine storm in COVID-19 patients.

\begin{tabular}{|c|c|c|c|c|c|c|c|c|c|c|c|}
\hline Study ID Number & Database & Intervention & Country & CRP (mg/L) & Ferritin $(\mu \mathrm{g} / \mathrm{L})$ & $\begin{array}{l}\text { D-Dimer } \\
\text { (ng/mL) }\end{array}$ & LDH (IU/L) & $\begin{array}{l}\text { Lymphocyte } \\
\text { (Cells } / \mu \mathrm{L})\end{array}$ & IL-6 (pg/mL) & Other Biomarkers & Fever \\
\hline NCT04443881 & $\begin{array}{l}\text { CT.gov, WHO } \\
\text { ICTRP }\end{array}$ & Anakinra & Spain & No & $>500$ & No & $>300$ & No & $>40$ & No & No \\
\hline NCT04356937 & CT.gov & Tocilizumab & United States & $>50$ & $>500$ & $>1000$ & $>250$ & No & No & No & Yes \\
\hline NCT04361526 & $\begin{array}{l}\text { CT.gov, WHO } \\
\text { ICTR }\end{array}$ & $\begin{array}{c}\text { Cytokine } \\
\text { Adsorption }\end{array}$ & Spain & $>10$ & No & No & No & No & No & No & No \\
\hline NCT04335071 & CT.gov & Tocilizumab & Switzerland & $\geq 50$ & No & No & No & No & No & No & No \\
\hline $\begin{array}{l}\text { 2020-001500-41; } \\
\text { EUCTR2020-001500- } \\
\text { 41-BE }\end{array}$ & $\begin{array}{c}\text { EU CTR, WHO } \\
\text { ICTRP }\end{array}$ & $\begin{array}{c}\text { Tocilizumab, } \\
\text { siltuximab, } \\
\text { anakinra }\end{array}$ & Belgium & $\begin{array}{l}>70 \text { and rising } \\
\text { since last } 24 \mathrm{~h}\end{array}$ & $\begin{array}{c}>1000 \text { and rising } \\
\text { since last } 24 \mathrm{~h} \\
>2000 \text { in patients } \\
\text { requiring } \\
\text { immediate high } \\
\text { flow oxygen } \\
\text { device or } \\
\text { mechanical } \\
\text { ventilation } \\
\text { if lymphopenia } \\
\text { and additional } \\
\text { criteria }>700 \text { and } \\
\text { rising since last } \\
24 \mathrm{~h}\end{array}$ & $\begin{array}{l}>1000 \text { and rising } \\
\text { since last } 24 \mathrm{~h}\end{array}$ & $>300$ & $<800$ & No & No & No \\
\hline NCT04394182 & $\begin{array}{l}\text { CT.gov, WHO } \\
\text { ICTRP }\end{array}$ & Radiotherapy & Spain & $\begin{array}{l}\text { Above normal } \\
\text { range }\end{array}$ & $\begin{array}{c}\text { Above the } \\
\text { normal range }\end{array}$ & $\begin{array}{l}\text { Above normal } \\
\text { range }\end{array}$ & $\begin{array}{l}\text { Above normal } \\
\text { range }\end{array}$ & $\begin{array}{l}\text { Below normal } \\
\text { range }\end{array}$ & $\begin{array}{l}\text { Above normal } \\
\text { range }\end{array}$ & Fibrinogen & No \\
\hline NCT04357860 & $\begin{array}{l}\text { CT.gov, WHO } \\
\text { ICTRP }\end{array}$ & Sarilumab & Spain & No & No & $\begin{array}{c}>1500 \text { or }>1000 \\
\text { if progressive } \\
\text { increases are } \\
\text { documented }\end{array}$ & No & No & $>40$ & No & No \\
\hline NCT04356690 & CT.gov & Etoposide & United States & $>100$ & $\begin{array}{l}>1000 \text { or }>500 \\
\text { with an } \\
\text { additional } \\
\text { biomarker }\end{array}$ & $>1000$ & $>500$ & No & No & WBC & No \\
\hline NCT04348383 & $\begin{array}{l}\text { CT.gov, WHO } \\
\text { ICTRP }\end{array}$ & Defibrotide & Spain & No & No & No & No & No & $\begin{array}{l}\geq 3 \times \text { upper } \\
\text { normal limit }\end{array}$ & No & No \\
\hline NCT04345445 & $\begin{array}{l}\text { CT.gov, WHO } \\
\text { ICTRP }\end{array}$ & $\begin{array}{l}\text { Tocilizumab, } \\
\text { methylpred- } \\
\text { nisolone }\end{array}$ & Malaysia & $\begin{array}{c}>60 \text { or an } \\
\text { increase }>20 \\
\text { over } 12 \mathrm{~h}\end{array}$ & Increasing & No & No & Declining & No & No & No \\
\hline
\end{tabular}


Table 2. Cont.

\begin{tabular}{|c|c|c|c|c|c|c|c|c|c|c|c|}
\hline Study ID Number & Database & Intervention & Country & CRP (mg/L) & Ferritin $(\mu \mathrm{g} / \mathrm{L})$ & $\begin{array}{l}\text { D-Dimer } \\
\text { (ng/mL) }\end{array}$ & LDH (IU/L) & $\begin{array}{l}\text { Lymphocyte } \\
\text { (Cells/ } / \mu \mathrm{L})\end{array}$ & IL-6 $(\mathrm{pg} / \mathrm{mL})$ & Other Biomarkers & Fever \\
\hline $\begin{array}{l}\text { 2020-001255-40; } \\
\text { EUCTR2020-001255- } \\
\text { 40-ES }\end{array}$ & $\begin{array}{l}\text { EU CTR, WHO } \\
\text { ICTRP }\end{array}$ & Sarilumab & Spain & $\begin{array}{c}>100 \text { or } \\
\text { increasing over } \\
24 \mathrm{~h}\end{array}$ & $>300$ & $\begin{array}{c}>1500 \text { or } \\
\text { progressive } \\
\text { increase (over } 3 \\
\text { consecutive } \\
\text { measurements) } \\
\text { and reaching } \\
\geq 1000\end{array}$ & No & $<800$ & No & No & No \\
\hline 2020-001375-32 & EU CTR & Tocilizumab & Netherlands & No & $\begin{array}{l}>2000 \text { or } \\
\text { doubling in } \\
20-48 \mathrm{~h}\end{array}$ & No & No & No & No & No & No \\
\hline NCT04403685 & CT.gov, EU CTR & Tocilizumab & Brazil & $>50$ & $>300$ & $>1000$ & $\begin{array}{c}\text { >upper level } \\
\text { limit }\end{array}$ & No & No & No & No \\
\hline RPCEC00000311 & WHO ICTRP & Itolizumab & Cuba & No & $\begin{array}{l}\text { Increased initial } \\
\text { value from } 500 \\
\text { or absolute } \\
\text { value } \geq 2000 \text {. }\end{array}$ & Increase & No & No & No & $\begin{array}{c}\text { Hemoglobin, } \\
\text { platelets, neutrophils, } \\
\text { ESR in mismatch } \\
\text { with CRP, } \\
\text { triglycerides, ALT, } \\
\text { Fibrinogen }\end{array}$ & Yes \\
\hline NCT04322773 & CT.gov & $\begin{array}{l}\text { Tocilizumab, } \\
\text { sarilumab }\end{array}$ & Denmark & $\begin{array}{c}>70 \text { or } \geq 40 \text { and } \\
\text { doubled within } \\
48 \mathrm{~h}\end{array}$ & 300 & $>1000$ & $>250$ & $<600$ & No & Platelet & No \\
\hline NCT04423042 & $\begin{array}{l}\text { CT.gov, WHO } \\
\text { ICTRP }\end{array}$ & Tocilizumab & Canada & $\geq 70$ & $\begin{array}{c}>700 \text { and/or } \\
\text { rising since last } \\
24 \mathrm{~h}\end{array}$ & No & No & No & No & No & No \\
\hline ChiCTR2000030196 & WHO ICTRP & Tocilizumab & China & No & No & No & No & No & Elevated & No & No \\
\hline $\begin{array}{r}\text { NCT04339712; } \\
\text { 2020-001039-29 }\end{array}$ & $\begin{array}{l}\text { CT.gov, EU CTR, } \\
\text { WHO ICTRP }\end{array}$ & $\begin{array}{c}\text { Tocilizumab, } \\
\text { anakinra }\end{array}$ & Greece & No & $>4420$ & No & No & No & No & No & No \\
\hline DRKS00021447 & WHO ICTRP & CytoSorb & Germany & $>100$ & No & No & No & No & No & No & No \\
\hline NCT04343963 & CT.gov & Pyridostigmine & Mexico & $>30$ & $>300$ & $>1000$ & $>245$ & $<800$ & No & Creatinine Kinase & No \\
\hline 2020-001390-76 & EU CTR & Sarilumab & Italy & $>30$ & $>500$ & $>1000$ & $>300$ & $<1000$ & No & No & No \\
\hline NCT04377503 & $\begin{array}{l}\text { CT.gov, WHO } \\
\text { ICTRP }\end{array}$ & $\begin{array}{l}\text { Tocilizumab, } \\
\text { methylpred- } \\
\text { nisolone }\end{array}$ & Brazil & $>50$ & $>300$ & $>1500$ & $>245$ & No & $>7$ & No & No \\
\hline $\begin{array}{l}\text { NCT04327505; } \\
\text { 2020-001349-37 }\end{array}$ & $\begin{array}{l}\text { CT.gov, EU CTR, } \\
\text { WHO ICTRP }\end{array}$ & Hyperbaric oxygen & $\begin{array}{l}\text { Germany, } \\
\text { Sweden }\end{array}$ & No & No & $>1000$ & No & No & No & No & No \\
\hline
\end{tabular}


Table 2. Cont.

\begin{tabular}{|c|c|c|c|c|c|c|c|c|c|c|c|}
\hline Study ID Number & Database & Intervention & Country & CRP (mg/L) & Ferritin $(\mu \mathrm{g} / \mathrm{L})$ & $\begin{array}{l}\text { D-Dimer } \\
\text { (ng/mL) }\end{array}$ & LDH (IU/L) & $\begin{array}{l}\text { Lymphocyte } \\
\text { (Cells } / \mu \mathrm{L})\end{array}$ & IL-6 (pg/mL) & Other Biomarkers & Fever \\
\hline NCT04359654 & $\begin{array}{l}\text { CT.gov, WHO } \\
\text { ICTRP }\end{array}$ & $\begin{array}{l}\text { Dornase alfa } \\
\text { inhalation }\end{array}$ & $\begin{array}{c}\text { United } \\
\text { Kingdom }\end{array}$ & $\geq 30$ & No & No & No & No & No & No & No \\
\hline NCT04397497 & CT.gov & Mavrilimumab & Italy & $\geq 60$ & $\geq 1000$ & No & $\begin{array}{l}\text { Above normal } \\
\text { range }\end{array}$ & No & No & No & Yes \\
\hline NCT04424056 & CT.gov & $\begin{array}{c}\text { Tocilizumab, } \\
\text { anakinra, } \\
\text { ruxolitinib }\end{array}$ & France & $>150$ & $>5000$ & No & No & No & No & No & No \\
\hline NCT04382755 & CT.gov & Zilucoplan & Belgium & $\begin{array}{l}>70 \text { and rising } \\
\text { since last } 24 \mathrm{~h}\end{array}$ & $\begin{array}{c}>1000 \text { and rising } \\
\text { since last } 24 \mathrm{~h} \\
>2000 \text { in patients } \\
\text { requiring } \\
\text { Optiflow or } \\
\text { mechanical } \\
\text { ventilation } \\
>700 \text { ug/L and } \\
\text { rising since last } \\
24 \mathrm{~h} \text { if } \\
\text { lymphopenia } \\
\text { and additional } \\
\text { criteria }\end{array}$ & $\begin{array}{l}>1000 \text { and rising } \\
\text { since last } 24 \mathrm{~h}\end{array}$ & $>300$ & $<800$ & No & No & No \\
\hline NCT04330638 & $\begin{array}{l}\text { CT.gov, WHO } \\
\text { ICTRP }\end{array}$ & $\begin{array}{c}\text { Tocilizumab, } \\
\text { anakinra, } \\
\text { siltuximab }\end{array}$ & Belgium & $\begin{array}{l}>70 \text { and rising } \\
\text { since last } 24 \mathrm{~h}\end{array}$ & $\begin{array}{c}>1000 \text { and rising } \\
\text { since last } 24 \mathrm{~h} \\
>2000 \text { in patients } \\
\text { requiring } \\
\text { Optiflow or } \\
\text { mechanical } \\
\text { ventilation } \\
>700 \text { ug/L and } \\
\text { rising since last } \\
24 \mathrm{~h} \text { if } \\
\text { lymphopenia } \\
\text { and additional } \\
\text { criteria }\end{array}$ & $\begin{array}{l}>1000 \text { and rising } \\
\text { since last } 24 \mathrm{~h}\end{array}$ & $\begin{array}{l}>300 \text { and } \\
\text { rising last } 24 \mathrm{~h}\end{array}$ & $<800$ & No & No & No \\
\hline $\begin{array}{l}\text { NCT04324021; } \\
\text { 2020-001167-93 }\end{array}$ & $\begin{array}{l}\text { CT.gov, EU CTR, } \\
\text { WHO ICTRP }\end{array}$ & $\begin{array}{c}\text { Emapalumab, } \\
\text { anakinra }\end{array}$ & Italy & No & $>500$ & $>1000$ & $>300$ & $<1000$ & No & No & No \\
\hline NCT04381052 & CT.gov & Clazakizumab & United States & $>35$ & $>500$ & $>1000$ & $>200$ & No & No & $\begin{array}{c}\text { Troponin } \\
\text { neutrophil- } \\
\text { lymphocyte } \\
\text { ratio }\end{array}$ & No \\
\hline NCT04343989 & CT.gov & Clazakizumab & United States & $>35$ & $>500$ & $>1000$ & $>200$ & No & No & $\begin{array}{c}\text { Troponin } \\
\text { neutrophil- } \\
\text { lymphocyte } \\
\text { ratio }\end{array}$ & No \\
\hline
\end{tabular}


Table 2. Cont.

\begin{tabular}{|c|c|c|c|c|c|c|c|c|c|c|c|}
\hline Study ID Number & Database & Intervention & Country & CRP (mg/L) & Ferritin $(\mu \mathrm{g} / \mathrm{L})$ & $\begin{array}{l}\text { D-Dimer } \\
\text { (ng/mL) }\end{array}$ & LDH (IU/L) & $\begin{array}{l}\text { Lymphocyte } \\
\text { (Cells } / \mu \mathrm{L})\end{array}$ & IL-6 (pg/mL) & Other Biomarkers & Fever \\
\hline NCT04363502 & CT.gov & Clazakizumab & United States & $>35$ & $>500$ & $>1000$ & $>200$ & No & No & $\begin{array}{c}\text { Troponin } \\
\text { neutrophil- } \\
\text { lymphocyte } \\
\text { ratio }\end{array}$ & No \\
\hline NCT04359290 & CT.gov & Ruxolitinib & Germany & No & $\begin{array}{l}\text { Above normal } \\
\text { value }\end{array}$ & No & $>283$ & No & No & No & No \\
\hline $\begin{array}{l}\text { NCT04362813; } \\
\text { 2020-001370-30 }\end{array}$ & $\begin{array}{l}\text { CT.gov, EU CTR, } \\
\text { WHO ICTRP }\end{array}$ & Canakinumab & $\begin{array}{l}\text { United States, } \\
\text { France, } \\
\text { Germany, } \\
\text { Italy, Russia, } \\
\text { Spain, United } \\
\text { Kingdom }\end{array}$ & $\geq 20$ & $\geq 600$ & No & No & No & No & No & No \\
\hline NCT04351243 & CT.gov & Gimsilumab & United States & Elevated & Elevated & No & No & No & No & No & No \\
\hline NCT04517162 & $\begin{array}{l}\text { CT.gov, WHO } \\
\text { ICTRP }\end{array}$ & $\begin{array}{c}\text { Collagen- } \\
\text { polyvinylpyrrolidone }\end{array}$ & United States & No & $>300$ & $>1000$ & No & $<800$ & No & $\begin{array}{l}\text { Creatinine kinase, } \\
\text { troponin }\end{array}$ & No \\
\hline NCT04470531 & CT.gov & Co-trimoxazole & Bangladesh & $>50$ & No & No & No & No & No & No & No \\
\hline NCT04560205 & CT.gov & Tocilizumab & Pakistan & $>50$ & $>1000$ & $>1000$ & $>1000$ & No & No & No & No \\
\hline NCT04559113 & CT.gov & Methylprednisolone & Pakistan & $>20$ & $>500$ & $>500$ & $>600$ & No & No & No & No \\
\hline NCT04528888 & CT.gov & Methylprednisolone & Italy & $\begin{array}{l}>6 \times \text { upper } \\
\text { normal limit }\end{array}$ & No & $\begin{array}{c}>6 \times \text { upper limit } \\
\text { of normal }\end{array}$ & No & No & No & No & No \\
\hline NCT04457349 & $\begin{array}{l}\text { CT.gov, WHO } \\
\text { ICTRP }\end{array}$ & $\begin{array}{c}\text { Therapeutic } \\
\text { Plasma Exchange }\end{array}$ & Egypt & Persistent high & No & No & No & No & $\begin{array}{l}\text { Persistent } \\
\text { high }\end{array}$ & No & Yes \\
\hline 2020-001645-40 & EU CTR & Reparixin & Italy & $\geq 30$ & $\geq 900$ & No & Elevated & No & $\geq 40$ & $\begin{array}{c}\text { Cross-linked fibrin } \\
\text { degradation } \\
\text { products }\end{array}$ & No \\
\hline 2020-001748-24 & EU CTR & $\begin{array}{l}\text { Tocilizumab, } \\
\text { anakinra }\end{array}$ & Sweden & $>70$ & $>500$ & $>500$ & $>470$ & $<1000$ & No & No & No \\
\hline $\begin{array}{l}\text { NCT04324021; } \\
\text { 2020-001167-93 }\end{array}$ & $\begin{array}{l}\text { CT.gov, EU CTR, } \\
\text { WHO ICTRP }\end{array}$ & $\begin{array}{c}\text { Emapalumab, } \\
\text { anakinra }\end{array}$ & Italy & No & $>500$ & $>1000$ & $>300$ & $<1000$ & No & No & No \\
\hline NCT04511819 & CT.gov & Losmapimod & $\begin{array}{l}\text { United States, } \\
\text { Brazil, Mexico }\end{array}$ & $>15$ & No & No & No & No & No & No & No \\
\hline
\end{tabular}

CT.gov: CliniclTrials.gov; EU CTR: European Union Clinical Trials registry; WHO ICTRP: World Health Organization International Clinical Trials Registry Platform. 
Table 3. Summary of specified criteria that were used in the different included studies.

\begin{tabular}{ccccccccccc}
\hline Criteria & $\begin{array}{c}\text { CRP } \\
(\mathbf{m g} / \mathbf{L})\end{array}$ & $\begin{array}{c}\text { Ferritin } \\
(\mu \mathrm{gg} / \mathrm{L})\end{array}$ & $\begin{array}{c}\text { D-Dimer } \\
(\mathbf{n g} / \mathbf{m L})\end{array}$ & $\begin{array}{c}\text { LDH } \\
(\mathbf{I U} / \mathbf{L})\end{array}$ & $\begin{array}{c}\text { Lymphocyte } \\
(\text { Cells/ } / \mu \mathrm{L})\end{array}$ & $\begin{array}{c}\text { IL-6 } \\
(\mathbf{p g} / \mathbf{m L})\end{array}$ & $\begin{array}{c}\text { Other } \\
\text { Biomarkers }\end{array}$ & $\begin{array}{c}\text { Fever } \\
\mathbf{O}_{2} / \text { Respiratory } \\
\text { Criteria }\end{array}$ & $\begin{array}{c}\text { Radiologic } \\
\text { Criteria }\end{array}$ \\
\hline $\begin{array}{l}\text { Number of } \\
\text { studies }(\%)\end{array}$ & $34(72 \%)$ & $35(74 \%)$ & $26(55 \%)$ & $24(51 \%)$ & $14(30 \%)$ & $8(17 \%)$ & $9(23 \%)$ & $\begin{array}{c}6 \\
(18 \%)\end{array}$ & $34(89 \%)$ & $29(62 \%)$ \\
\hline
\end{tabular}

A serum ferritin measurement was required in 35 trials (74\%), CRP in 34 trials (72\%), D-dimer in 26 trials (55\%), LDH in 24 trials (51\%), lymphocyte count in 14 trials (30\%), and IL-6 in 8 trials (17\%). There were 11 studies that included other biomarkers (platelets, WBCs, transaminases, fibrinogen, cross-linked fibrin degradation products, neutrophillymphocyte ratio, troponin, creatinine kinase, triglycerides, and hemoglobin). The most common cutoff values for ferritin were $>500 \mu \mathrm{g} / \mathrm{L}$ (11 studies), $>300 \mu \mathrm{g} / \mathrm{L}$ ( 6 studies), $>1000 \mu \mathrm{g} / \mathrm{L}$ ( 6 studies), and $>2000 \mu \mathrm{g} / \mathrm{L}$ ( 5 studies). For CRP, the most common cutoff values were $>30-35 \mathrm{mg} / \mathrm{L}$ ( 7 studies), $>70 \mathrm{mg} / \mathrm{L}$ ( 6 studies), $>50 \mathrm{mg} / \mathrm{L}$ ( 5 studies), and $>100 \mathrm{mg} / \mathrm{L}$ ( 3 studies). The most common D-dimer cutoff values were $>1000 \mathrm{ng} / \mathrm{mL}$ (18 studies), $>500 \mathrm{ng} / \mathrm{mL}$ ( 3 studies), and $>1500 \mathrm{ng} / \mathrm{mL}$ (3 studies). For lymphocyte counts, the most common cutoff values were $<500$ cells $/ \mu \mathrm{L}$ ( 5 studies) and $<1000$ cells $/ \mu \mathrm{L}$ ( 5 studies). The most common LDH cutoff values were $>300 \mathrm{IU} / \mathrm{L}$ (7 studies), $>245-250 \mathrm{IU} / \mathrm{L}$ (4 studies), and $>200$ IU/L ( 3 studies). For IL- 6 , the most common cutoff value was $>40 \mathrm{pg} / \mathrm{mL}$ (3 studies).

\section{Discussion}

CRS is a systemic inflammatory response caused by the release of inflammatory cytokines, such as IL-6, interferon gamma (IFN $\gamma$ ), tumor necrosis factor-alpha (TNF $\alpha$ ), IL-2, and IL-10. This is the result of the activation of a large number of lymphocytes (B cells, $\mathrm{T}$ cells, and/or natural killer cells) and/or myeloid cells (macrophages, dendritic cells, and monocytes). CRS can manifest with a constellation of clinical symptoms including fever, hypotension, and widespread organ dysfunction [14]. There is no international consensus on unified criteria for the diagnosis of CRS. In addition, cytokine elevation was thought to be a late finding in patients with COVID-19 [15].

Although CRS is increasingly described in patients with severe or critical COVID-19, our review demonstrates that there is significant variability in the definition and defining criteria for CRS in the included prospective trials. Most of the included studies involved patients who meet the severe to critical case definition of COVID-19, as per the American National Institutes of Health (NIH) [1]. This finding implies that CRS occurrence is limited to those with severe and critical cases. Several grading scales for the severity of CRS have been suggested. Originally, CAR T cell therapy-induced CRS was categorized according to the Common Terminology Criteria for Adverse Events version 4.03 (CTCAEv4.03), released in 2009. This grading scale was used to classify CRS-related adverse events caused by immunotherapies [16]. Since then, several attempts were made to develop a more concise grading scale. In 2014, Lee et al. modified the CRS grading, as per CTCAEv4.03, to define mild, moderate, severe, and life-threatening CRS regardless of the inciting agent [14]. Another published rating scale for CRS was devised by Davila et al., in 2015 [17]. In 2018, Neelapu et al. proposed a grading scale for CRS very similar to the Lee criteria [18]. In March 2018, CTCAE v5.0 was published with significantly modified grading criteria for CRS that account for patients' responses to fluids, oxygen requirements, need for vasopressors, and organ dysfunction [19]. Recently, the American Society for Blood and Marrow Transplantation sponsored a meeting to come to a consensus for CRS and CARassociated neurotoxicity grading [20]. In the present review, all but one $(46 / 47)$ of the prospective trials included clinical manifestations in their inclusion criteria, with hypoxia being the most commonly included. This finding is in contrast to what was found in the above-mentioned grading scales of CRS, where fever and hypotension were the most common included criteria. It is important to realize that most of the CRS cases examined 
during the development of these grading scales were secondary to CAR T cell and other immunotherapies, which may provoke more profound cytokine release and systemic inflammation. In our review, the CRS patients included in these trials are COVID-19 patients, and as such, hypoxia may be attributed to viral pneumonia rather than the development of CRS per se. Regarding the radiographic investigations, 29 studies out of $47(62 \%)$ mandated the presence of radiologic changes in chest X-rays or CT scans and thus, the contribution of radiological changes to the diagnosis of CRS is not a unified criterion for the diagnosis of CRS. Radiographic features of ARDS are the most commonly described changes in CRS. However, the sensitivity and specificity of these changes are not known yet.

We could not elucidate any specific biochemical biomarker for an accurate diagnosis of CRS. All of the included studies mandated the presence of laboratory abnormality. There are several inflammatory markers to be considered with this condition, including CRP, ferritin, D-dimer, LDH, IL-6, and lymphocyte count. However, serum ferritin and CRP are the most selected biomarkers ( $74 \%$ and $72 \%$, respectively). The sensitivity and specificity of these biomarkers for CRS are yet to be evaluated. Other biomarkers included in these trials were fibrinogen, cross-linked fibrin degradation products, platelets, hemoglobin, WBCs, creatinine kinase, triglycerides, liver enzymes, and troponin. As mentioned above, CRS is associated with multi-organ involvement and hence, many biochemical abnormalities might be present. Moreover, it seems that there are no clear diagnostic cutoffs for these biomarkers in CRS. We found significant variations in the cutoff levels of these biomarkers among the included studies. Davila et al. added cytokine elevation as a criterion for severe CRS, defined as at least a 75-fold elevation of two serum cytokines over baseline, or a 250-fold elevation of at least one serum cytokine over baseline [17]. Additionally, they identified 7 cytokines, of the 39 measured, whose elevation strongly correlated with CRS. These cytokines were IFN- $\gamma$, IL5, IL6, IL10, Flt-3L, Fractalkine, and GM-CSF [17].

In a recent rapid systematic review, Leisman DE et al. compared the maximum levels of several inflammatory biomarkers (TNF $\alpha$, IL-8, IL-1 $\beta$, IL-10, IL-2, IL-4, soluble IL-2 receptor, IFN $\gamma, \mathrm{CRP}$, ferritin, fibrinogen, D-dimer, LDH, ESR, albumin, procalcitonin, total bilirubin, lymphocyte count, and platelet count) between COVID-19-induced CRS and three other systemic inflammatory conditions: sepsis, non-COVID-19-related ARDS, and non-COVID-19-induced CRS [21]. The calculated mean IL-6 level in COVID-19 patients was $36.7 \mathrm{pg} / \mathrm{mL}$ (ranging between 6.5 and $357.2 \mathrm{pg} / \mathrm{mL}$ ). The mean IL-6 serum level was nearly 100 times higher in patients with CAR T cell-induced CRS compared with patients with COVID-19. Similarly, the pooled mean IL-6 level was much higher in patients with hyperinflammatory ARDS $(1558.2 \mathrm{pg} / \mathrm{mL})$ and patients with sepsis $(983.6 \mathrm{pg} / \mathrm{mL})$ compared with COVID-19 patients. Patients with COVID-19 had substantially higher D-dimer elevations than did patients with sepsis. Most other cytokines were comparatively low in COVID-19 patients [21]. These data may explain why anti-cytokine therapy has not been universally effective for the treatment of severe COVID-19 in several randomized clinical trials [1]. However, larger-scale trials are warranted to further explore the role of anti-cytokine therapy and immunomodulators in severe COVID-19 infection, considering their efficacy, safety, and patient characteristics [22,23]. In our systematic review, most trials used monoclonal antibodies, with tocilizumab, followed by anakinra, being the most studied.

It is believed that CRS is a major contributor to increased morbidity and mortality in COVID-19 patients [2]. Currently, the options to control this disease and its complications are quite limited. The use of immune modulators in COVID-19-associated CRS shows some promising results [24]. With a better understanding of CRS and its pathophysiological aspects in COVID-19 infection, more focused efforts can take place to find the proper treatment of COVID-19 patients. This understanding will also help to guide the timing of immune modulator therapy administration, its dosing, and if repeat courses are indicated. 


\section{Conclusions}

There is high variability in CRS definitions and associated biomarker cutoff values in the COVID-19 clinical trials. We call to form a unified definition of CRS, especially in COVID-19 patients, as this is a critical step to move forward in designing COVID-19 therapeutics. Such a unified definition would be an important contribution to the scientific community. However, having an expert consensus on such a definition is beyond the scope of this review and deserves international collaborative future work.

Author Contributions: Conceptualization, K.E., H.M. and J.A.A.-T.; methodology, K.E., H.M., M.B. and J.A.A.-T.; formal analysis, K.E., S.A. and M.A.; investigation, K.E., S.A. and M.A.; data curation, K.E.; writing—original draft preparation, K.E., H.M., S.A., M.A. and M.B.; writing-review and editing, J.A.A.-T.; visualization, K.E., H.M., S.A., M.A., J.A.A.-T.; supervision, K.E.; project administration, K.E. All authors have read and agreed to the published version of the manuscript.

Funding: This research received no external funding.

Conflicts of Interest: The authors declare no conflict of interest.

\section{Appendix A}

Table A1. $\mathrm{O}_{2} /$ respiratory criteria and radiologic criteria in the included studies describing cytokine storm in COVID-19 patients.

\begin{tabular}{|c|c|c|}
\hline Study ID Number & $\mathrm{O}_{2} /$ Respiratory Criteria & Radiologic Criteria \\
\hline NCT04443881 & $\begin{array}{c}\mathrm{SpO} 2 \leq 94 \% \text { measured with a pulse oximeter } \\
\mathrm{Pa}: \mathrm{FiO} 2 \leq 300 \\
\mathrm{Sa}: \mathrm{FiO} 2 \leq 350\end{array}$ & $\begin{array}{l}\text { CXR (or other technique) pulmonary } \\
\text { infiltrates compatible with pneumonia }\end{array}$ \\
\hline NCT04356937 & $\begin{array}{l}\text { O2 supplementation not }>10 \text { L delivered by any device } \\
\text { Need for supplemental O2 to maintain saturation }>92 \%\end{array}$ & Pulmonary infiltrate on CXR \\
\hline NCT04361526 & $\begin{array}{c}\text { Worsening respiratory symptoms } \\
\mathrm{PaO} 2: \mathrm{FiO} 2<200 \mathrm{mmHg} \\
\text { Pulmonary wedge pressure }<18 \mathrm{mmHg} \\
\mathrm{RR}>30\end{array}$ & $\begin{array}{l}\text { Bilateral pulmonary infiltrates on } \\
\text { chest imaging }\end{array}$ \\
\hline NCT04335071 & $\begin{array}{c}\mathrm{SpO} 2<93 \% \\
\mathrm{PaO} 2<65 \mathrm{mmHg} \\
\text { Persistent or increasing } \mathrm{O} 2 \text { demand or dyspnea } \\
\mathrm{RR} \geq 25\end{array}$ & $\begin{array}{l}\text { Radiographic evidence compatible } \\
\text { with pneumonia }\end{array}$ \\
\hline $\begin{array}{l}\text { 2020-001500-41; } \\
\text { EUCTR2020-001500- } \\
\quad \text { 41-BE }\end{array}$ & $\begin{array}{c}\mathrm{PaO} 2 / \mathrm{FiO} 2<350 \text { while breathing room air in upright } \\
\text { position or } \mathrm{PaO} 2 / \mathrm{FiO} 2<280 \text { on supplemental oxygen and } \\
\text { immediately requiring high flow oxygen device or } \\
\text { mechanical ventilation }\end{array}$ & $\begin{array}{l}\text { CXR and/or CT scan showing bilateral } \\
\text { infiltrates within last } 2 \text { days }\end{array}$ \\
\hline NCT04394182 & $\begin{array}{c}\mathrm{SpO} 2<93 \% \\
\text { Oxygen therapy escalation (Understanding from less to } \\
\text { more need for support: Nasal Cannula; Ventimask } \pm \\
\text { reservoir) } \\
\mathrm{Pa} 02 / \mathrm{Fi} 02<300 \mathrm{mmHg}\end{array}$ & $\begin{array}{c}\text { Worsening of total severity score } \\
\text { throughout admission or score at } \\
\text { admission }>5 \text { by a diagnostic baseline } \\
\text { CT scan }\end{array}$ \\
\hline $\begin{array}{l}\text { NCT04366232; } \\
\text { 2020-001963-10 }\end{array}$ & $\begin{array}{l}\mathrm{RR}>30, \mathrm{PaO} 2<90 \mathrm{mmHg} \\
\text { ARDS defined by a patient under mechanical ventilation } \\
\text { with a } \mathrm{PaO} 2 / \mathrm{FiO} 2<300 \text { for }>24 \mathrm{~h}\end{array}$ & No \\
\hline NCT04357860 & $\begin{array}{c}\text { Absence of ARDS requiring ONAF or } \\
\text { mechanical ventilation }\end{array}$ & $\begin{array}{l}\text { Interstitial pneumonia confirmed by } \\
\text { chest radiography or CT }\end{array}$ \\
\hline NCT04356690 & $\begin{array}{c}\text { Intubated or requiring }>4 \mathrm{~L} / \text { min of supplemental } \mathrm{O} 2 \text { to } \\
\text { maintain } \mathrm{SpO} 2>92 \% \text { without intubation }\end{array}$ & No \\
\hline
\end{tabular}


Table A1. Cont.

\begin{tabular}{|c|c|c|}
\hline Study ID Number & $\mathrm{O}_{2} /$ Respiratory Criteria & Radiologic Criteria \\
\hline NCT04397497 & $\begin{array}{l}\text { Requiring } \mathrm{O} 2 \text { supplementation }(\mathrm{SpO} 2 \leq 92 \%) \text { and having a } \\
\text { PAO2/FIO2 } \leq 300 \mathrm{mmHg}\end{array}$ & $\begin{array}{l}\text { Pneumonia evidenced by CXR or CT } \\
\text { with pulmonary infiltrates }\end{array}$ \\
\hline NCT04348383 & Requiring respiratory support & No \\
\hline NCT04345445 & $\begin{array}{c}\text { Dyspnoea OR RR }>20 \text { breaths } / \text { min AND O2 sat }<93 \% \text { on } \\
\text { RA OR increasing need for O2 supplementation to maintain } \\
\text { O2 sat }>95 \% \text { on RA }\end{array}$ & $\begin{array}{l}\text { CXR or CT indicative of pneumonia OR } \\
\text { worsening findings over time }\end{array}$ \\
\hline $\begin{array}{l}\text { 2020-001255-40; } \\
\text { EUCTR2020-001255- } \\
\quad 40-E S\end{array}$ & High oxygen requirements & Evidence of pneumonia \\
\hline 2020-001375-32 & Hypoxia & No \\
\hline NCT04403685 & $\begin{array}{l}\text { Need for oxygen supplementation to keep SPO2 }>93 \% \text { or } \\
\text { need for mechanical ventilation for less than } 24 \mathrm{~h}\end{array}$ & CT with COVID-19 alterations \\
\hline RPCEC00000311 & $\begin{array}{l}\text { Need for oxygen therapy to maintain } \mathrm{SpO} 2>93 \% \\
\text { Worsening of lung involvement, defined as one of the } \\
\text { following criteria: (a) worsening } \mathrm{SpO} 2>3 \% \text { or decrease in } \\
\mathrm{PaO} 2>10 \% \text {, with } \mathrm{FiO} 2 \text { stable in the last } 24 \mathrm{~h} \text {, (b) need to } \\
\text { increase } \mathrm{FiO} 2 \text { in order to maintain a stable } \mathrm{SO} 2 \text { or new need } \\
\text { for mechanical installation in the last } 24 \mathrm{~h} \text {, (c) increase in the } \\
\text { number and/or extent of consolidation lung areas }\end{array}$ & $\begin{array}{l}\text { Multifocal interstitial pneumonia and } \\
\text { worsening of the radiological image }\end{array}$ \\
\hline NCT04322773 & $\begin{array}{l}\text { Need for } \mathrm{O} 2 \text { therapy to maintain } \mathrm{SpO} 2>94 \% \text { or } \mathrm{FiO} 2 / \mathrm{PaO} 2 \\
\qquad>20\end{array}$ & $\begin{array}{c}\text { Consolidation, ground glass opacities, or } \\
\text { bilateral pulmonary infiltration by CT } \\
\text { or CXR }\end{array}$ \\
\hline NCT04362111 & No & No \\
\hline NCT04423042 & No & No \\
\hline ChiCTR2000030196 & No & Severe pneumonia \\
\hline $\begin{array}{l}\text { NCT04339712; } \\
\text { 2020-001039-29 }\end{array}$ & No & No \\
\hline DRKS00021447 & No & No \\
\hline NCT04343963 & $\begin{array}{c}\text { Dyspnoea } \\
\mathrm{Pa}: \mathrm{FiO} 2<300 \mathrm{mmHg} \\
\mathrm{SpO} 2<90 \% \text {, or a } 3 \% \text { drop in baseline oximetry, or need to } \\
\text { increase supplemental O2 due to chronic hypoxia, as well as } \\
\text { the need for supplemental O2 }\end{array}$ & $\begin{array}{l}\text { Pneumonia confirmed by imaging studies } \\
\text { with increased mortality criteria such as } \\
\text { lung infiltrates }>50 \% \text { of lung fields by CT }\end{array}$ \\
\hline 2020-001390-76 & $\begin{array}{c}\mathrm{SpO} 2 \text { without } \mathrm{O} 2 \text { supplementation }<93 \% \text { or } \mathrm{PaO} 2 / \mathrm{FiO} 2< \\
300 \text { in patients requiring } \mathrm{O} 2 \text { supplementation }\end{array}$ & $\begin{array}{l}\text { Evidence of pulmonary infiltrates at CT } \\
\text { or CXR }\end{array}$ \\
\hline NCT04377503 & $\mathrm{Pao} 2 / \mathrm{FIO} 2<200$ & No \\
\hline $\begin{array}{l}\text { NCT04327505; } \\
\text { 2020-001349-37 }\end{array}$ & $\mathrm{PaO} 2 / \mathrm{FiO} 2<200 \mathrm{mmHg}$ & No \\
\hline NCT04359654 & $\mathrm{SpO} 2 \geq 94 \%$ on supplementary $\mathrm{O} 2$ & No \\
\hline NCT04424056 & $\begin{array}{c}\qquad \mathrm{RR}>30 / \mathrm{min}, \mathrm{PaO} 2<90 \mathrm{mmHg} \\
\text { ARDS (mechanically ventilated patient with } \mathrm{PaO} 2 / \mathrm{FiO} 2< \\
\qquad 300 \text { for }>24 \mathrm{~h} \\
\text { Moderate to severe ARDS }(\mathrm{PaO} 2 / \mathrm{FiO} 2<200 \text { to } \mathrm{PEEP} \text { of at } \\
\text { least } 8 \mathrm{cmH} 2 \mathrm{O} \text { ) on invasive mechanical ventilation }\end{array}$ & COVID-19 pneumonia \\
\hline NCT04382755 & $\begin{array}{c}\mathrm{PaO} 2 / \mathrm{FiO} 2<350 \text { or } \mathrm{PaO} 2 / \mathrm{FiO} 2<280 \text { on supplemental } \mathrm{O} 2 \\
\text { and immediately requiring Optiflow or } \\
\text { mechanical ventilation }\end{array}$ & $\begin{array}{l}\text { CT showing bilateral infiltrates within } \\
\text { last } 2 \text { days }\end{array}$ \\
\hline
\end{tabular}


Table A1. Cont.

\begin{tabular}{|c|c|c|}
\hline Study ID Number & $\mathrm{O}_{2} /$ Respiratory Criteria & Radiologic Criteria \\
\hline NCT04330638 & $\begin{array}{c}\mathrm{PaO} 2 / \mathrm{FiO} 2<350 \text { or } \mathrm{PaO} 2 / \mathrm{FiO} 2<280 \text { on supplemental } \mathrm{O} 2 \\
\text { and immediately requiring Optiflow or mechanical } \\
\text { ventilation }\end{array}$ & $\begin{array}{l}\text { CXR or CT showing bilateral infiltrates } \\
\text { within last } 2 \text { days }\end{array}$ \\
\hline $\begin{array}{l}\text { NCT04324021; } \\
\text { 2020-001167-93 }\end{array}$ & $\begin{array}{c}\mathrm{PaO} 2 / \mathrm{FiO} 2<300 \mathrm{mmHg} \text { and }>200 \mathrm{~mm} \mathrm{Hg} \\
\mathrm{RR} \geq 30 \\
\mathrm{SpO} 2<93 \%\end{array}$ & No \\
\hline NCT04381052 & $\begin{array}{c}\mathrm{PaO} 2 / \mathrm{FiO} 2<200, \mathrm{SpO} 2<90 \% \text { on } 4 \mathrm{~L} \text {, or increasing } \mathrm{O} 2 \\
\text { requirements over } 24 \mathrm{~h}\end{array}$ & No \\
\hline NCT04343989 & $\begin{array}{c}\mathrm{PaO} 2 / \mathrm{FiO} 2<200, \mathrm{SpO} 2<90 \% \text { on } 4 \mathrm{~L} \text {, or increasing } \mathrm{O} 2 \\
\text { requirements over } 24 \mathrm{~h}\end{array}$ & No \\
\hline NCT04363502 & $\begin{array}{c}\mathrm{PaO} 2 / \mathrm{FiO} 2<200, \mathrm{SpO} 2<90 \% \text { on } 4 \mathrm{~L} \text {, or increasing } \mathrm{O} 2 \\
\text { requirements over } 24 \mathrm{~h}\end{array}$ & No \\
\hline NCT04359290 & $\begin{array}{c}\text { Recent intubation } \\
\mathrm{PaO} 2 / \mathrm{FiO} 2 \leq 200 \mathrm{mmHg} \text { at a PEEP } \geq 5 \mathrm{~mm} \mathrm{H} 2 \mathrm{O}\end{array}$ & CT: pulmonary infiltration \\
\hline $\begin{array}{l}\text { NCT04362813; } \\
\text { 2020-001370-30 }\end{array}$ & $\mathrm{SpO} 2 \leq 93 \%$ or $\mathrm{PaO} 2 / \mathrm{FiO} 2<300 \mathrm{mmHg}$ & $\begin{array}{l}\text { Pneumonia evidenced by CXR or CT } \\
\text { with pulmonary infiltrates }\end{array}$ \\
\hline NCT04351243 & $\begin{array}{c}\text { Subject requires high-flow oxygen or meets clinical } \\
\text { classification for ARDS }\end{array}$ & $\begin{array}{l}\text { Radiographic evidence of } \\
\text { bilateral infiltrates }\end{array}$ \\
\hline NCT04517162 & $\begin{array}{c}\mathrm{SpO} 2<92 \% \text { or requiring supplemental } \mathrm{O} 2 \text { or mechanical } \\
\text { ventilation }\end{array}$ & $\begin{array}{l}\text { Radiologic findings by imaging study: } \\
\text { inflammatory infiltrates }\end{array}$ \\
\hline NCT04470531 & $\mathrm{SpO} 2<90 \%$ or increasing $\mathrm{O} 2$ requirement & $\begin{array}{l}\text { Bilateral crackles on auscultation or CXR } \\
\text { with bilateral infiltrates }\end{array}$ \\
\hline NCT04560205 & $\begin{array}{l}\mathrm{SpO} 2 \leq 93 \% \\
\mathrm{RR}>30-35\end{array}$ & $\begin{array}{l}>50 \% \text { of radiological involvement of lung } \\
\text { with typical lesions }\end{array}$ \\
\hline NCT04559113 & $\mathrm{RR}>22$ & $\begin{array}{c}>50 \% \text { of radiological involvement of lung } \\
\text { with typical lesions }\end{array}$ \\
\hline
\end{tabular}

\begin{tabular}{|c|c|c|}
\hline NCT04528888 & $\begin{array}{l}\text { Positive pressure ventilation from }>24 \mathrm{~h} \\
\text { Invasive mechanical ventilation from }<96 \mathrm{~h} \\
\mathrm{PaO} 2 / \mathrm{FiO} 2<150 \mathrm{mmHg}\end{array}$ & No \\
\hline NCT04457349 & $\begin{array}{l}\text { Persistent worsening of respiratory symptoms } \\
\qquad \mathrm{PaO} 2 / \mathrm{FiO} 2<150 \mathrm{mmHg}\end{array}$ & No \\
\hline 2020-001645-40 & $\begin{array}{c}\text { Respiratory distress } \\
\mathrm{RR} \geq 30 \\
\mathrm{PaO} 2 / \mathrm{FiO} 2<300 \mathrm{mmHg}\end{array}$ & $\begin{array}{l}\text { Chest imaging confirms lung } \\
\text { involvement and inflammation. }\end{array}$ \\
\hline 2020-001748-24 & $5 \mathrm{~L} / \mathrm{min}$ of oxygen to maintain $\mathrm{SpO} 2$ at $\geq 93 \%$ & No \\
\hline $\begin{array}{l}\text { NCT04324021; } \\
\text { 2020-001167-93 }\end{array}$ & $\begin{aligned} \mathrm{PaO} 2 / \mathrm{FiO} 2 & <300 \mathrm{~mm} \mathrm{Hg} \\
\mathrm{RR} & \geq 30 \\
\mathrm{SpO} 2 & <93 \%\end{aligned}$ & COVID-19 pneumonia \\
\hline NCT04511819 & $\begin{array}{l}\mathrm{SpO} 2 \geq 90 \% \text { on room air and / or } \geq 94 \% \text { on oxygen } \\
\text { administration at } 2 \mathrm{~L} / \mathrm{min} \text { by nasal cannula }\end{array}$ & $\begin{array}{l}\text { Radiographic evidence of pulmonary } \\
\text { involvement consistent with COVID-19 }\end{array}$ \\
\hline
\end{tabular}

\section{References}

1. COVID-19 Treatment Guidelines Panel. Coronavirus Disease 2019 (COVID-19) Treatment Guidelines. National Institutes of Health. Available online: https:/ / www.covid19treatmentguidelines.nih.gov/ (accessed on 5 February 2021).

2. Huang, C.; Wang, Y.; Li, X.; Ren, L.; Zhao, J.; Hu, Y.; Zhang, L.; Fan, G.; Xu, J.; Gu, X.; et al. Clinical features of patients infected with 2019 novel coronavirus in Wuhan, China. Lancet 2020, 395, 497-506. [CrossRef] 
3. Al Sulaiman, K.A.; Aljuhani, O.; Eljaaly, K.; Alharbi, A.A.; Al Shabasy, A.M.; Alsaeedi, A.S.; Al Mutairi, M.; Badreldin, H.A.; Al Harbi, S.A.; Al Haji, H.A.; et al. Clinical features and outcomes of critically ill patients with coronavirus disease 2019 (COVID-19): A multicenter cohort study. Int. J. Infect. Dis. 2021, 105, 180-187. [CrossRef] [PubMed]

4. Tisoncik, J.R.; Korth, M.J.; Simmons, C.P.; Farrar, J.; Martin, T.R.; Katze, M.G. Into the eye of the cytokine storm. Microbiol. Mol. Biol. Rev. 2021, 76, 16-32. [CrossRef] [PubMed]

5. Shimabukuro-Vornhagen, A.; Gödel, P.; Subklewe, M.; Stemmler, H.J.; Schlößer, H.A.; Schlaak, M.; Kochanek, M.; Böll, B.; von Bergwelt-Baildon, M.S. Cytokine release syndrome. J. Immunother. Cancer 2018, 6, 1-14. [CrossRef]

6. Sinha, P.; Matthay, M.A.; Calfee, C.S. Is a "cytokine storm" relevant to COVID-19? JAMA Intern. Med. 2020, 180, 1152-1154. [CrossRef]

7. Channappanavar, R.; Perlman, S. Pathogenic human coronavirus infections: Causes and consequences of cytokine storm and immunopathology. Semin. Immunopathol. 2017, 39, 529-539. [CrossRef]

8. Chatenoud, L.; Ferran, C.; Legendre, C.; Thouard, I.; Merite, S.; Reuter, A.; Kreis, H.; Franchimont, P.; Bach, J.F. In vivo cell activation following OKT3 administration. Systemic cytokine release and modulation by corticosteroids. Transplantation 1990, 49, 697-702. [CrossRef]

9. Gauthier, J.; Turtle, C.J. Insights into cytokine release syndrome and neurotoxicity after CD19-specific CAR-T cell therapy. Curr. Res. Transl. Med. 2018, 66, 50-52. [CrossRef]

10. Klinger, M.; Brandl, C.; Zugmaier, G.; Hijazi, Y.; Bargou, R.C.; Topp, M.S.; Gökbuget, N.; Neumann, S.; Goebeler, M.; Viardot, A.; et al. Immunopharmacologic response of patients with B-lineage acute lymphoblastic leukemia to continuous infusion of T cell-engaging CD19/CD3-bispecific BiTE antibody blinatumomab. Blood J. Am. Soc. Hematol. 2012, 119, 6226-6233. [CrossRef] [PubMed]

11. Hay, K.A.; Hanafi, L.A.; Li, D.; Gust, J.; Liles, W.C.; Wurfel, M.M.; López, J.A.; Chen, J.; Chung, D.; Harju-Baker, S.; et al. Kinetics and biomarkers of severe cytokine release syndrome after CD19 chimeric antigen receptor-modified T-cell therapy. Blood 2017, 130, 2295-2306. [CrossRef] [PubMed]

12. Kantarjian, H.; Stein, A.; Gökbuget, N.; Fielding, A.K.; Schuh, A.C.; Ribera, J.M.; Wei, A.; Dombret, H.; Foà, R.; Bassan, R.; et al. Blinatumomab versus chemotherapy for advanced acute lymphoblastic leukemia. N. Engl. J. Med. 2017, 376, 836-847. [CrossRef]

13. Mehta, P.; McAuley, D.F.; Brown, M.; Sanchez, E.; Tattersall, R.S.; Manson, J.J. COVID-19: Consider cytokine storm syndromes and immunosuppression. Lancet 2020, 395, 1033-1034. [CrossRef]

14. Lee, D.W.; Gardner, R.; Porter, D.L.; Louis, C.U.; Ahmed, N.; Jensen, M.; Grupp, S.A.; Mackall, C.L. Current concepts in the diagnosis and management of cytokine release syndrome. Blood 2014, 124, 188-195. [CrossRef]

15. England, J.T.; Abdulla, A.; Biggs, C.M.; Lee, A.; Hay, K.A.; Hoiland, R.L.; Wellington, C.L.; Sekhon, M.; Jamal, S.; Shojania, K.; et al. Weathering the COVID-19 storm: Lessons from hematologic cytokine syndromes. Blood Rev. 2021, 45, 100707. [CrossRef] [PubMed]

16. US Department of Health and Human Services. Common Terminology Criteria for Adverse Events (CTCAE); V4.03; National Institutes of Health: Bethesda, MD, USA, 2010.

17. Davila, M.L.; Riviere, I.; Wang, X.; Bartido, S.; Park, J.; Curran, K.; Chung, S.S.; Stefanski, J.; Borquez-Ojeda, O.; Olszewska, M.; et al. Efficacy and toxicity management of 19-28z CAR T cell therapy in B cell acute lymphoblastic leukemia. Sci. Transl. Med. 2014, 6, 224ra25. [CrossRef] [PubMed]

18. Neelapu, S.S.; Tummala, S.; Kebriaei, P.; Wierda, W.; Gutierrez, C.; Locke, F.L.; Komanduri, K.V.; Lin, Y.; Jain, N.; Daver, N.; et al. Chimeric antigen receptor T-cell therapy-assessment and management of toxicities. Nat. Rev. Clin. Oncol. 2018, 15, 47-62. [CrossRef]

19. US Department of Health and Human Services. Common Terminology Criteria for Adverse Events (CTCAE); V5.0; National Institutes of Health: Bethesda, MD, USA, 2018.

20. Lee, D.W.; Santomasso, B.D.; Locke, F.L.; Ghobadi, A.; Turtle, C.J.; Brudno, J.N.; Maus, M.V.; Park, J.H.; Mead, E.; Pavletic, S.; et al. ASTCT Consensus Grading for Cytokine Release Syndrome and Neurologic Toxicity Associated with Immune Effector Cells. Biol. Blood Marrow Transplant. J. Am. Soc. Blood Marrow Transplant. 2019, 25, 625-638. [CrossRef]

21. Leisman, D.E.; Ronner, L.; Pinotti, R.; Taylor, M.D.; Sinha, P.; Calfee, C.S.; Hirayama, A.V.; Mastroiani, F.; Turtle, C.J.; Harhay, M.O.; et al. Cytokine elevation in severe and critical COVID-19: A rapid systematic review, meta-analysis, and comparison with other inflammatory syndromes. Lancet Respir. Med. 2020, 8, 1233-1244. [CrossRef]

22. Eldanasory, O.A.; Eljaaly, K.; Memish, Z.A.; Al-Tawfiq, J.A. Histamine release theory and roles of antihistamine in the treatment of cytokines storm of COVID-19. Travel Med. Infect. Dis. 2020, 37, 101874. [CrossRef]

23. Eljaaly, K.; Alireza, K.H.; Alshehri, S.; Al-Tawfiq, J.A. Hydroxychloroquine safety: A meta-analysis of randomized controlled trials. Travel Med. Infect. Dis. 2020, 36, 101812. [CrossRef]

24. Zhong, J.; Tang, J.; Ye, C.; Dong, L. The immunology of COVID-19: Is immune modulation an option for treatment? Lancet Rheumatol. 2020, 2, e428-e436. [CrossRef] 